\title{
Desenvolvimento de equações Intensidade-Duração-Frequência sem dados pluviográficos em regiões semiáridas
} \author{
Eunice M. de Andrade ${ }^{4}$ \& Paulo R. L. Tavares ${ }^{5}$ \\ ${ }^{1}$ UFCA. Juazeiro do Norte, CE. E-mail: vicentefechine@hotmail.com \\ ${ }^{2}$ UFCA. Juazeiro do Norte, CE. E-mail: joseison@cariri.ufc.br \\ ${ }^{3}$ UFCA. Juazeiro do Norte, CE. E-mail: larm@.ufc.br (Autor correspondente) \\ ${ }^{4}$ UFC. Fortaleza, CE. E-mail: eandrade@ufc.br \\ ${ }^{5}$ UFCA. Juazeiro do Norte, CE. E-mail: prltavares@ufc.br
}

Vicente Fechine Sobrinho ${ }^{1}$, Joseilson O. Rodrigues ${ }^{2}$, Luiz A. R. Mendonça ${ }^{3}$,

\section{Palavras-chave:}

chuvas intensas

isozonas

desagregação

distribuição de probabilidade

\begin{abstract}
R E S U M O
Neste estudo foram feitas a validação da metodologia de desagregação de chuvas das isozonas e sua utilização no desenvolvimento de equações IDF (Intensidade-Duração-Frequência) para três municípios da Região Metropolitana do Cariri, Ceará, desprovidos de pluviógrafos. Na validação utilizaram-se dados de estações localizadas em zonas climatológicas distintas da Região Metropolitana do Cariri e de uma estação localizada na mesma zona. As precipitações máximas diárias anuais foram ajustadas e testadas pelas distribuições de probabilidade de Gumbel, Log-Normal II-P, Log-Normal III-P, Gama II-P e Gama III-P. As intensidades de chuvas estimadas pelo método das isozonas apresentaram altos índices de concordância e desempenho de Willmott, quando comparadas com as estimadas pelas respectivas equações IDF. As precipitações máximas diárias anuais utilizadas na aplicação do método das isozonas se ajustaram, respectivamente, às distribuições Gama III, Log-Normal II e Gama II; entretanto, verificou-se que outras funções podem ser utilizadas sem perda significativa na precisão das estimativas; esses resultados indicam a viabilidade da utilização do método das isozonas como alternativa na elaboração das equações IDF em regiões não monitoradas.
\end{abstract}

Key words: intense rains isozones disaggregation probability distribution

\section{Development of Intensity-Duration-Frequency equation without rainfall data in semiarid regions}

\begin{abstract}
A B S T R A C T
The isozones rain disaggregation methodology was validated and used in this study for the development of IDF (Intensity-Duration-Frequency) equations for three municipalities in the Cariri Metropolitan Region - Ceará, devoid of recording rain gauges. For the validation, data from stations located in different climate regions of the Cariri Metropolitan Region, and from one station in the same climate region was used. To develop the IDF equations, annual daily maximum rainfall data were used, which were adjusted and tested according to Gumbel, Log-Normal II-P, Log-Normal III-P, Gama II-P e Gama III-P probability distributions. The rainfall intensity estimated by the isozones method presented high Willmott indexes, when compared to the amount estimated by the respective IDF equations. The maximum annual daily rainfall used in the application of the isozones method, adjusted respectively to the Gamma III, Normal-Log II and Gamma II. However, it was verified that other functions may be used without significant loss to the precision of the estimates. These results indicate the viability of using this method as an alternative to elaborate IDF equations for regions not monitored and without records of rainfall.
\end{abstract}

\section{INTRODUÇÃO}

A Região Metropolitana do Cariri, localizada no semiárido do Nordeste, ao Sul do Estado do Ceará, é uma área de intenso crescimento econômico e populacional, com cerca de $80 \%$ da população concentrada em uma zona adjacente englobando três municípios principais: Juazeiro do Norte, Crato e Barbalha. Devido ao caráter convectivo das chuvas aliado ao intenso crescimento desordenado, essa região vem sendo constantemente afetada por eventos extremos de precipitações sendo necessário, portanto, o desenvolvimento de equações Intensidade-Duração-Frequência (IDF) locais; todavia, a falta de informações pluviográficas tem conduzido ao uso de equações desenvolvidas para municípios localizados fora desta região. Segundo Genovez \& Zuffo (2000) esta prática pode levar a erros de projeto haja vista que essas equações refletem peculiaridades climáticas específicas de cada região.

As características das chuvas intensas, mais especificamente de modelos que associam Intensidade, Duração e Frequência (IDF), permitem um planejamento mais seguro na 
determinação de estruturas de contenção do processo erosivo (Martins et al., 2010) além de possibilitar o dimensionamento de estruturas hidráulicas para canalização de escoamento superficial, dentre outras utilizações (Genovez \& Zuffo, 2000; Rodrigues et al., 2008; Santos et al., 2010; Alhassoun, 2011; Back et al., 2012; Elsebaie, 2012); contudo, para o desenvolvimento das equações IDF, normalmente é necessário dispor de uma grande base de dados pluviográficos que, na maioria das vezes, não estão acessíveis devido à inexistência de uma rede contínua de monitoramento ou de um número reduzido de informações disponíveis, como é o caso da região em estudo.

Ante a baixa disponibilidade de séries históricas adequadas, uma alternativa seria a estimativa das intensidades a partir de dados pluviométricos com validação e utilização criteriosa de modelos de desagregação de chuvas (Svensson et al., 2007; Garcia et al., 2011).

Esta possibilidade se origina, por exemplo, a partir de estudos realizados que mostram a existência de relações constantes entre os totais precipitados em um dia e os totais precipitados em períodos mais curtos, conforme metodologia das isozonas proposta por Torrico (1974). O método se baseia na desagregação de precipitações máximas diárias em chuvas de durações diferentes de $24 \mathrm{~h}$ fundamentando-se na delimitação de zonas de mesma relação pluviométrica $1 \mathrm{~h} / 24$ $\mathrm{h}$ e $6 \mathrm{~min} / 24 \mathrm{~h}$, para um período de retorno base de um ano. Relações pluviométricas referentes a outros períodos de retorno são obtidas dividindo-se as do período de retorno base pelo coeficiente empírico de Pfafstetter (Torrico, 1974). As zonas de comportamento semelhante são identificadas construindo gráficos que correlacionam linearmente a duração em escala logarítmica com a respectiva chuva intensa diária em escala aritmética permitindo também determinar chuvas para outras durações.

Para minimizar os efeitos da escassez de informações ou do número reduzido de registros de intensidades de chuvas, tem-se buscado alternativas para obter chuvas de menores durações a partir de dados pluviométricos diários. As metodologias descritas na literatura utilizam, em sua maioria, coeficientes de conversão de chuvas de $24 \mathrm{~h}$ em durações menores (Svensson et al., 2007; Oliveira et al., 2008; Back, 2009; Fechine Sobrinho, 2011). Entretanto, a simples utilização deste método sem uma análise mais rigorosa ou verificação por meio de validação, pode levar a erros. Diante disto objetivou-se, neste estudo, validar e aplicar a metodologia de desagregação de chuvas das isozonas no desenvolvimento de equações IDF para três municípios da Região Metropolitana do Cariri, CE (RMC) desprovidos de dados pluviógráficos.

\section{MATERIAL E Métodos}

$\mathrm{Na}$ validação do método das isozonas (Torrico, 1974) utilizaram-se registros pluviográficos das estações de Fortaleza, CE (Estação Funceme, ANA - n 338048) com 23 anos de dados, Miracema do Norte, TO (Estação Miracema do Tocantins, ANA - no 948000) com informações de 19 anos e registros pluviográficos da estação de Aiuaba, CE, monitorada pelo Grupo de Pesquisas Hidrossedimentológicas do Semiárido (Estação BEA, $06^{\circ} 42^{\prime} 17^{\prime \prime} \mathrm{S}$ e $40^{\circ} 17^{\prime} 17^{\prime \prime} \mathrm{W}$ ) (11 anos). Foram utilizados, também, registros pluviométricos da estação de Aiuaba, CE (Estação Aiuaba, ANA - nº 640019) com informações de 79 anos.

As equações IDF foram elaboradas para as estações de Juazeiro do Norte, Crato e Barbalha, localizados na Região Metropolitana do Cariri, ao sul do Estado do Ceará, entre as coordenadas geográficas $6^{\circ} 23^{\prime} 42^{\prime \prime}$ e $6^{\circ} 23^{\prime} 47^{\prime \prime} \mathrm{S}$ e $39^{\circ} 15^{\prime} 24^{\prime \prime}$ e $39^{\circ} 15^{\prime} 29^{\prime \prime}$ W (Figura 1). O clima é classificado como BSwh', clima semiárido com chuvas máximas de outono. Das estações desses três municípios utilizaram-se registros pluviométricos pertencentes ao banco de informações da Fundação Cearense de Meteorologia (FUNCEME) com período de observação de 37 anos (1974- 2010).

As precipitações diárias máximas anuais $\left(\mathrm{P}_{1 \text { dia }}\right)$ estimadas pela distribuição de probabilidade ajustada foram convertidas em chuvas virtuais de $24 \mathrm{~h}\left(\mathrm{P}_{24 \mathrm{~h}}\right)$ utilizando-se a Eq. 1. Segundo Back (2009) as chuvas máximas medidas em um dia diferem das máximas de $24 \mathrm{~h}$ em virtude das leituras diárias serem realizadas sempre no mesmo horário podendo, em alguns eventos ininterruptos, ser inferiores ao que deveria ser registrado em $24 \mathrm{~h}$.

$$
\mathrm{P}_{24 \mathrm{~h}}=1,096 \times \mathrm{P}_{\text {ldia }}
$$

Posteriormente, identificou-se a zona de comportamento semelhante utilizando-se o sistema de informações geográficas aplicado ao mapa de isozonas proposto por Torrico (1974) de onde se obtiveram as relações pluviométricas $1 \mathrm{~h} / 24 \mathrm{~h} \mathrm{e} 6 \mathrm{~min} / 24$ $\mathrm{h}$ utilizadas no cálculo das chuvas de duração de 1 h e 6 min apenas multiplicando-se pela chuva virtual de $24 \mathrm{~h}$.

Correlacionando linearmente as durações de $6 \mathrm{~min}, 1$ h e 24 $\mathrm{h}$ em escala logarítmica e as respectivas chuvas virtuais intensas em escala aritmética para dado período de retorno, obtiveram-se as chuvas correspondentes a outras durações. As intensidades foram avaliadas para as durações de 6, 12, 18, 24,30 e 36 min e $1 ; 1,5 ; 2 ; 3$ e $4 \mathrm{~h}$, correspondentes aos períodos de retornos de $5,10,15,20,25,30,50$ e100 anos.

A validação do método das isozonas (Torrico, 1974) foi feita para duas estações meteorológicas localizadas em zonas climatológicas distintas da RMC e uma na mesma zona.

Para as zonas climatológicas distintas foram utilizadas equações IDF desenvolvidas criteriosamente por Laura (1997) a partir de dados pluviográficos com período de observação de 23 anos, para a estação de Fortaleza, CE (isozona C) caracterizada como de influência marítima (Eq. 2) e de 19 anos para a estação de Miracema do Norte, TO (isozona E, caracterizada como continental e noroeste) (Eqs. 3 e 4). A distribuição de probabilidade utilizada na obtenção dessas equações foi a Gama III.

$$
\mathrm{I}=\frac{36,16 \times(\operatorname{Tr}+1)^{0,154}}{(\mathrm{t}+26,94)^{0,864}}, \text { para } 5 \min \leq \mathrm{t} \leq 240 \leq \min
$$




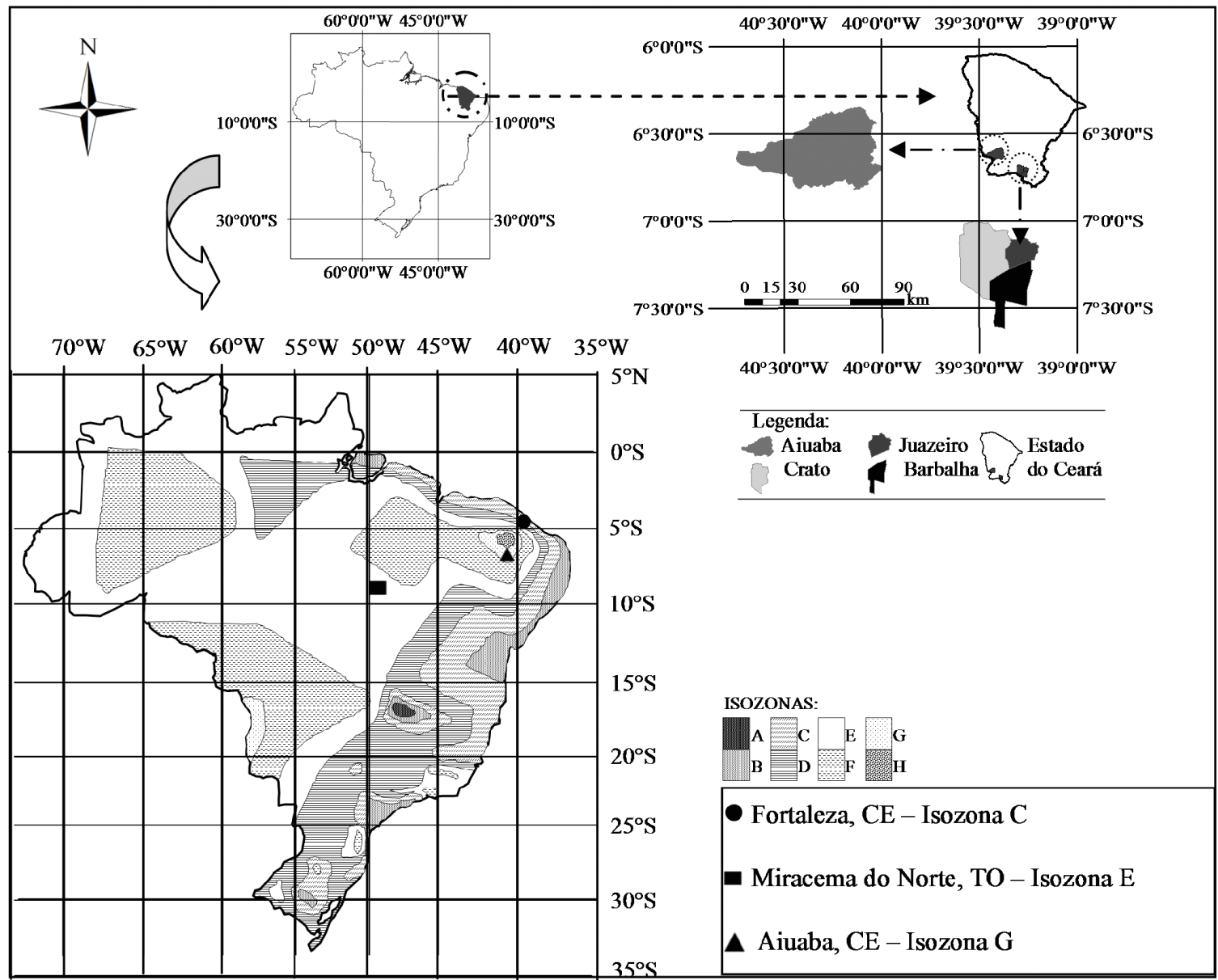

Figura 1. Localização das estações de estudo e isozonas

$$
\begin{gathered}
I=\frac{28,99 \times(\operatorname{Tr}+1)^{0,101}}{(t+17)^{0,796}}, \text { para } \mathrm{t} \leq 45 \mathrm{~min} \\
I=\frac{16,6 \times(\operatorname{Tr}+1)^{0,134}}{\mathrm{t}^{0,737}}, \text { para } \mathrm{t}>45 \mathrm{~min}
\end{gathered}
$$

Nesta validação utilizou-se, com nível de significância 5\%, o teste ANOVA para as equações de regressão a fim de verificar se a relação linear entre os valores calculados pelas equações IDF e os estimados pelo método das isozonas é estatisticamente significante e o teste t para analisar se esta relação linear é do tipo $\mathrm{Y}=\mathrm{X}$, ou seja, se as hipóteses de que o coeficiente angular da reta é igual a 1 (um) e o intercepto $Y$ é igual a 0 não podem ser rejeitadas a nível de significância 0,05 . Adicionalmente, foi utilizada a interpretação dos índices de concordância (d) (Eq. 5) e de desempenho (c) de Willmott (Camargo \& Sentelhas, 1997).

$$
\mathrm{d}=\left(1-\frac{\sum\left(\mathrm{O}_{\mathrm{i}}-\mathrm{E}_{\mathrm{i}}\right)}{\sum\left(/ \mathrm{O}_{\mathrm{i}}-\mathrm{O}_{\mathrm{m}} /\right)+\left(/ \mathrm{E}_{\mathrm{i}}-\mathrm{E}_{\mathrm{m}} /\right)}\right) \times 100
$$

em que:

$\mathrm{O}_{\mathrm{i}}$ e $\mathrm{E}_{\mathrm{i}}$ - intensidades calculadas pela equação IDF e estimadas pelo método das isozonas, respectivamente

$\mathrm{O}_{\mathrm{m}}$ e $\mathrm{E}_{\mathrm{m}}$ - médias das intensidades calculadas e estimadas, respectivamente

O índice d varia de zero, para ausência de concordância, a um, para concordância perfeita. O índice c é obtido pelo produto entre o coeficiente de correlação e o índice d e também varia de zero a um, com $c>0,85$ para desempenho ótimo, $c \leq 0,40$ para péssimo e intervalos de classes intermediárias de c (Camargo \& Sentelhas, 1997).

Para a zona climatológica semelhante à da RMC (isozona G, caracterizada como de caatinga nordestina) foram comparadas as relações pluviométricas $1 \mathrm{~h} / 24 \mathrm{~h}$ e $6 \mathrm{~min} / 24 \mathrm{~h}$, obtidas da estação de Aiuaba, CE, com as estabelecidas por Torrico (1974). Nesta análise utilizaram-se dados pluviométricos e pluviográficos recentes correspondentes a um período de observação de onze anos (de 2003 a 2013). A partir dos registros pluviométricos máximos diários e utilizando-se a distribuição Gumbel, estimou-se uma altura pluviométrica para o período de retorno de um ano, que é o tempo base descrito no método para obtenção dessas relações. A partir dos registros pluviográficos selecionou-se, dentre os eventos diários, a lâmina precipitada correspondente ao valor estimado pela distribuição. Assim, foram extraídas as precipitações correspondentes às durações de $6 \mathrm{~min}, 1 \mathrm{~h}$ e 24 h, que é a base do método das isozonas.

No ajuste aos modelos estatísticos utilizados na elaboração da equação IDF utilizou-se a distribuição Gumbel como ponto de partida para estimativa dos eventos extremos (Oliveira et al., 2008; Rodrigues et al., 2008; Garcia et al., 2011) e em seguida 
as distribuições teóricas de probabilidade Log-Normal II-P, Log-Normal III-P, Gama II-P e Gama III-P, descritas por Back (2001) e Catalhunha et al. (2002). As distribuições ajustadas foram utilizadas na obtenção das precipitações máximas anuais possíveis de ocorrer em dado período de retorno.

Para avaliar o ajuste das distribuições de probabilidades utilizaram-se os testes de aderência qui-quadrado $\left(\chi^{2}\right)$ e Lilliefors, associados aos índices estatísticos desvio quadrático médio (DQM) (Eq. 6) e desvio percentual médio absoluto (DPMA) (Eq. 7) e aos coeficientes angular e de determinação da reta de correlação entre as precipitações máximas diárias anuais observadas e estimadas.

$$
\begin{gathered}
D Q M=\sqrt{\frac{1}{N} \sum_{i=1}^{N} \frac{\left(\mathrm{xo}_{\mathrm{i}}-\mathrm{xc}_{\mathrm{i}}\right)^{2}}{\mathrm{xc}_{\mathrm{i}}}} \\
\text { DPMA }=\frac{1}{\mathrm{~N}} \sum_{\mathrm{i}=1}^{\mathrm{N}} \frac{\left|\mathrm{xo}_{\mathrm{i}}-\mathrm{xc}_{\mathrm{i}}\right|}{\mathrm{xc}_{\mathrm{i}}}
\end{gathered}
$$

em que:

$$
\begin{array}{ll}
\mathrm{Xo}_{\mathrm{i}} & \text { - I-ésima precipitação observada } \\
\mathrm{Xc}_{\mathrm{i}} & \text { - I-ésima precipitação calculada } \\
\mathrm{N} & \text { - tamanho da amostra }
\end{array}
$$

Para cada uma das estações da RMC foram obtidas, após obtenção das intensidades pelo método das isozonas, equações IDF, conforme Eq. 8.

$$
I=\frac{a(\operatorname{Tr}+s)^{b}}{(t+c)^{n}}
$$

em que:

I - intensidade máxima da chuva, $\mathrm{mm} \mathrm{min}^{-1}$

$\operatorname{Tr} \quad$ - período de retorno, anos

$\mathrm{t}$ - duração da chuva, min

a, b, c, n e s - parâmetros empíricos para cada local

O parâmetro $\mathrm{c}$ foi estimado conforme a metodologia descrita por Wilken (1978) (Eq. 8). De acordo com este método foram relacionados, para um período de retorno de cinco anos, três pares ordenados de intensidades e durações $\left(\mathrm{I}_{1}, \mathrm{t}_{1}\right),\left(\mathrm{I}_{2}, \mathrm{t}_{2}\right)$ e $\left(\mathrm{I}_{3}\right.$, $t_{3}$ ), em que, $I_{1}$ e $I_{2}$ - Intensidades correspondentes às durações de $5 \min \left(\mathrm{t}_{1}\right)$ e $240 \mathrm{~min}\left(\mathrm{t}_{2}\right) ; \mathrm{I}_{3}$ - é produto $\sqrt{\mathrm{I}}_{1}$. $\sqrt{\mathrm{I}_{2}}$. A duração $\mathrm{t}_{3}$ é obtida a partir de $\mathrm{I}_{3}$, diretamente da série gerada pela metodologia das isozonas.

$$
c=\frac{\left(t_{3}^{2}-t_{1} \times t_{2}\right)}{\left(t_{1}+t_{2}-2 \times t_{3}\right)}
$$

Os parâmetros a, b e $\mathrm{n}$ foram determinados através de análise de regressão linear múltipla aplicada à Eq. 10 (obtida da Eq. 8) utilizando-se o método dos mínimos quadrados.

$$
\operatorname{Ln}(\mathrm{I})=\operatorname{Ln}(\mathrm{a})+\mathrm{b} \times \operatorname{Ln}(\operatorname{Tr}+\mathrm{s})-\mathrm{n} \times \operatorname{Ln}(\mathrm{t}+\mathrm{C})
$$

Os parâmetros foram obtidos minimizando o $\chi^{2}$ dado por uma função polinomial do tipo $\chi^{2}=\mathrm{f}(\mathrm{s})$, obtida por ajuste aos pontos $\left(s, \chi^{2}\right)$. Neste contexto o $\chi^{2}$ compara as séries de intensidades estimadas pelo método das isozonas com as calculadas pela Eq. 8 em função de s, para cada conjunto de parâmetros a, b, n e C; assim se terá, para cada s, um $\chi^{2}$ e o valor de s que minimizará o $\chi^{2}$ otimizará a Eq. 8. Com um $\chi^{2}$ mínimo aumenta-se a probabilidade de aceitação da hipótese de nulidade obtendo-se um valor de s ótimo.

\section{Resultados e Discussão}

Os bons ajustes apresentados pelos coeficientes de determinação, angular e linear (Figura 2A e B) para a estação de Fortaleza e a de Miracema do Norte-TO, sugerem que o

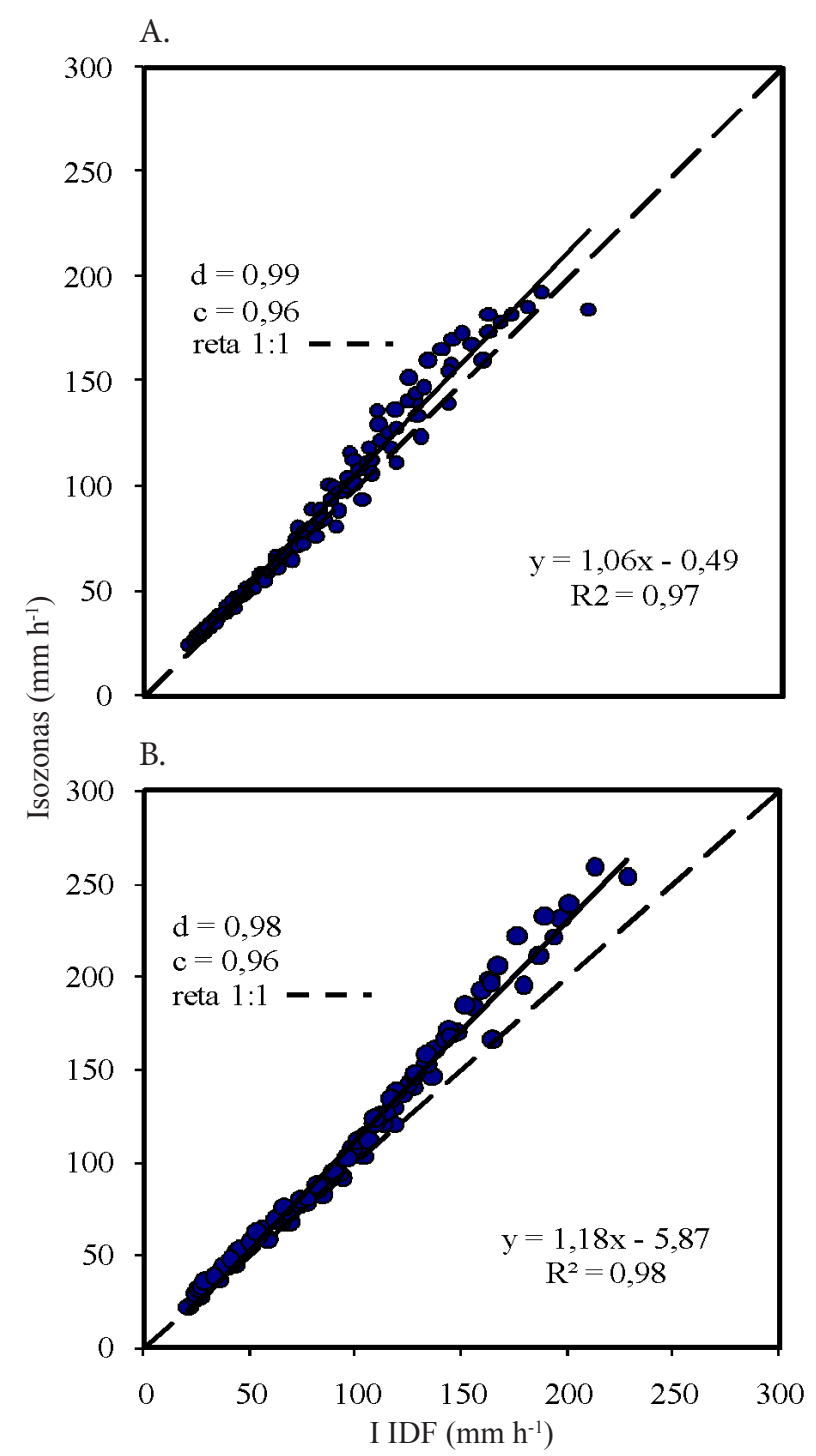

Figura 2. Correlação linear entre intensidades máximas calculadas pelas equações IDF de Laura (1997) (eixo y) e as estimadas pelo método das isozonas (eixo x), para as estações de Fortaleza, CE (A) e Miracema do Norte, TO (B). d e c são respectivamente os índices de concordância e de desempenho de Willmott 
método das isozonas reproduz adequadamente as intensidades calculadas pelas equações IDF.

A análise de variância dos modelos indica, com segurança, a ocorrência da relação linear entre os valores obtidos pelo método das isozonas e aqueles calculados pelas equações IDF; para isto foram comparados as estatísticas de teste $\left(\mathrm{F}_{0}\right)$ e os valores críticos $\left(\mathrm{F}_{c}\right)$, constatando-se que $\mathrm{F}_{0}>\mathrm{F}_{c}$ para as duas cidades: Fortaleza/CE $(3258,33$ > 3,94) e Miracema do Norte/ TO $(7550,49>3,94)$.

Os testes $t$ relacionados aos coeficientes das retas indicam que em ambas as cidades para valores de intensidade abaixo de $125 \mathrm{~mm} \mathrm{~h}^{-1}$, não se pode rejeitar a hipótese de que $\mathrm{Y}=\mathrm{X}$, ou seja, para este limite os resultados obtidos pelo método das isozonas e pelas equações IDF são equivalentes. Para valores acima de 125 $\mathrm{mm} \mathrm{h}^{-1} \mathrm{o}$ método das Isozonas tende a superestimar os valores calculados pelas equações IDF. Além do mais e de acordo com o índice de concordância (d) de Willmott essas correlações lineares apresentam alta confiabilidade com valores de 0,99 e 0,98 para as respectivas estações de Fortaleza, CE, e Miracema do Norte, TO. Em ambas os índices de desempenho (c) de Willmott também foram classificados ótimos, de conformidade com a avaliação de Camargo \& Sentelhas (1997).

A análise deste índice de concordância de Camargo reforça a hipótese de que a metodologia das isozonas pode ser utilizada nessas estações com expressiva confiabilidade na estimativa das intensidades máximas, como alternativa das chuvas de projeto e corroboram com os de Oliveira et al. (2008) que avaliaram o desempenho de diversos métodos indiretos de estimativa de intensidades máximas no estado de Goiás indicando o método das isozonas como o de melhor desempenho.
Os desvios entre as intensidades máximas calculadas pelas equações IDF e pelo método das isozonas, em Fortaleza, CE, e Miracema do Norte, TO, estão na Tabela 1. Para a estação de Fortaleza os valores absolutos dos desvios variaram de 0,1 a $22,9 \%$ sendo que o valor médio não ultrapassou $12,1 \%$; já para Miracema do Norte variaram de 0,4 e 20,2\%, com média inferior a $15,5 \%$.

De maneira geral verificou-se, na estação de Fortaleza, $\mathrm{CE}$, que aproximadamente $76 \%$ dos desvios entre os valores observados e estimados não excederam $10 \%$; já na estação de Miracema do Norte-TO, aproximadamente $90 \%$ dos desvios não ultrapassaram 15\%. Esta comparação indica uma aproximação aceitável na estimativa levando em consideração que se está estimando intensidades em local sem dados pluviográficos. Esses resultados reafirmam a premissa de adequação do método das isozonas para obtenção de séries sintéticas de intensidades para serem utilizadas na elaboração de pequenos projetos de engenharia (Fechine Sobrinho, 2011) ou dimensionamento de estrutura de contenção erosiva (Martins et al., 2010).

Uma provável explicação para aproximação entre os valores calculados pelas equações IDF e estimados pelo método das isozonas, constatada pelos baixos desvios, pode estar relacionada ao uso de séries de mesmo tamanho, duração e período de retorno. Genovez \& Zuffo (2000) alertam que a estimativa de intensidades em desacordo com os limites da equação IDF (tamanho, duração e período de retorno) pode incorrer em erros, superestimando ou subestimando os valores. Os desvios observados neste trabalho foram inferiores aos obtidos por Garcia et al. (2011) que avaliaram a utilização de duas metodologias de desagregação em estações no estado

Tabela 1. Desvios percentuais das intensidades máximas calculadas pela equação IDF e estimadas pelo método das isozonas, para diferentes durações e períodos de retorno, para as estações de Fortaleza e Miracema do Norte

\begin{tabular}{|c|c|c|c|c|c|c|c|c|c|c|c|c|}
\hline \multirow{2}{*}{$\begin{array}{l}\text { Período de retorno } \\
\text { (anos) }\end{array}$} & \multicolumn{12}{|c|}{ Durações (min) } \\
\hline & 6 & 12 & 18 & 24 & 30 & 36 & 48 & 60 & 90 & 120 & 180 & 240 \\
\hline & \multicolumn{12}{|c|}{ Estação de Fortaleza, CE } \\
\hline 5 & 10,2 & 22,9 & 18,9 & 14,9 & 11,8 & 9,4 & 6,3 & 4,3 & 6,9 & 8,8 & 11,5 & 13,5 \\
\hline 10 & 9,4 & 21,2 & 17,1 & 13,1 & 10,0 & 7,6 & 4,5 & 2,5 & 5,4 & 7,4 & 10,2 & 12,3 \\
\hline 15 & 8,2 & 19,5 & 15,4 & 11,3 & 8,3 & 5,9 & 2,8 & 0,9 & 3,8 & 5,9 & 8,8 & 10,9 \\
\hline 20 & 7,1 & 17,9 & 13,7 & 9,7 & 6,7 & 4,4 & 1,3 & $-0,7$ & 2,4 & 4,5 & 7,4 & 9,5 \\
\hline 25 & 6,1 & 16,7 & 12,5 & 8,5 & 5,4 & 3,2 & 0,1 & $-1,8$ & 1,3 & 3,4 & 6,3 & 8,4 \\
\hline 30 & 5,2 & 15,5 & 11,3 & 7,3 & 4,3 & 2,1 & $-1,0$ & $-2,9$ & 0,2 & 2,4 & 5,3 & 7,5 \\
\hline 50 & 2,5 & 12,0 & 7,8 & 3,9 & 0,9 & $-1,3$ & $-4,3$ & $-6,2$ & $-2,9$ & $-0,8$ & 2,3 & 4,4 \\
\hline 100 & $-11,8$ & 2,6 & 0,2 & $-2,8$ & $-5,1$ & $-6,9$ & $-9,4$ & $-11,0$ & $-7,6$ & $-5,4$ & $-2,4$ & $-0,3$ \\
\hline Máximo (absoluto) & 11,8 & 22,9 & 18,9 & 14,9 & 11,8 & 9,4 & 9,4 & 11,0 & 7,6 & 8,8 & 11,5 & 13,5 \\
\hline Mínimo (absoluto) & 2,5 & 2,6 & 0,2 & 2,8 & 0,9 & 1,3 & 0,1 & 0,7 & 0,2 & 0,8 & 2,3 & 0,3 \\
\hline \multirow[t]{2}{*}{ Média } & 7,5 & 16,0 & 12,1 & 8,9 & 6,6 & 5,1 & 3,7 & 3,8 & 3,8 & 4,8 & 6,8 & 8,3 \\
\hline & \multicolumn{12}{|c|}{ Estação de Miracema do Norte, TO } \\
\hline 5 & 0,4 & 5,4 & 2,3 & $-0,6$ & $-2,8$ & $-4,6$ & $-3,2$ & $-1,8$ & 2,5 & 4,4 & 5,6 & 5,7 \\
\hline 10 & 7,8 & 12,0 & 8,9 & 6,2 & 4,1 & 2,4 & 1,1 & 2,3 & 6,7 & 8,6 & 9,9 & 10,1 \\
\hline 15 & 11,1 & 14,7 & 11,7 & 9,0 & 6,9 & 5,2 & 2,5 & 3,7 & 8,2 & 10,2 & 11,5 & 11,8 \\
\hline 20 & 13,1 & 16,5 & 13,5 & 10,8 & 8,8 & 7,1 & 3,5 & 4,7 & 9,2 & 11,1 & 12,5 & 12,8 \\
\hline 25 & 14,4 & 17,5 & 14,5 & 11,9 & 9,8 & 8,1 & 3,8 & 5,0 & 9,6 & 11,5 & 13,0 & 13,3 \\
\hline 30 & 15,4 & 18,3 & 15,3 & 12,7 & 10,6 & 9,0 & 4,1 & 5,2 & 9,9 & 11,9 & 13,4 & 13,7 \\
\hline 50 & 17,7 & 20,2 & 17,2 & 14,6 & 12,5 & 10,9 & 4,4 & 5,5 & 10,3 & 12,4 & 14,0 & 14,3 \\
\hline 100 & 9,9 & 18,3 & 16,6 & 14,7 & 13,1 & 11,8 & 3,6 & 5,1 & 10,1 & 12,3 & 14,0 & 14,4 \\
\hline Máximo (absoluto) & 17,7 & 20,2 & 17,2 & 14,7 & 13,1 & 11,8 & 4,4 & 5,5 & 10,3 & 12,4 & 14,0 & 14,4 \\
\hline Mínimo (absoluto) & 0,4 & 4,8 & 2,3 & 0,6 & 2,8 & 2,4 & 1,1 & 1,4 & 2,5 & 2,7 & 2,8 & 2,9 \\
\hline Média & 11,2 & 15,4 & 12,5 & 10,1 & 8,6 & 7,4 & 3,3 & 4,2 & 8,3 & 10,3 & 11,7 & 12,0 \\
\hline
\end{tabular}

Desvios negativos indicam que os valores estimados subestimam os observados pela equação IDF 
do Mato Grosso comparando os valores estimados com os calculados pelas equações IDF.

Para validação da isozona $\mathrm{G}$, onde se encontram as estações da RMC, compararam-se as relações pluviométricas $1 \mathrm{~h} / 24$ h e $6 \mathrm{~min} / 24 \mathrm{~h}$, obtidas da estação de Aiuaba, CE, com as estabelecidas por Torrico (1974). Estimou-se, utilizando a distribuição de Gumbel a partir dos dados pluviométricos (79 anos) uma precipitação máxima diária de $37 \mathrm{~mm}$ para um período de retorno de um ano, que é o tempo base descrito no método para obtenção dessas relações. A partir dos dados pluviográficos disponíveis (11 anos) foram encontrados sete eventos com precipitações próximas à máxima estimada (37 $\mathrm{mm}$ ). Com esses eventos selecionados obtiveram-se as alturas pluviométricas para as durações de $1 \mathrm{~h}$ e 6 min e as relações 1 h/24 h e $6 \mathrm{~min} / 24 \mathrm{~h}$ (Tabela 2).

Tabela 2. Relações pluviométricas percentuais $1 \mathrm{~h} / 24 \mathrm{~h}$ e $6 \mathrm{~min} / 24 \mathrm{~h}$ e erro relativo (E) entre os valores da zona $\mathrm{G}$ e os calculados para Aiuaba, $\mathrm{CE}$, para o período de retorno de um ano

\begin{tabular}{ccc}
\hline & $\mathbf{1 ~ h / 2 4} \mathbf{~ h}$ & $\mathbf{6 ~} \mathbf{~} \mathbf{m} / \mathbf{2 4} \mathbf{~ h}$ \\
Método das isozonas ${ }^{(1)}$ & 49,0 & 15,4 \\
Aiuaba-CE & 48,8 & 15,4 \\
$\mathrm{E}(\%)$ & 0,4 & 0,0 \\
\hline
\end{tabular}

Os valores encontrados por Torrico (1974) para a isozona $\mathrm{G}$ apresentaram erro relativo inferior a $1 \%$ com relação aos coeficientes calculados neste estudo. Esses resultados indicam que o método das isozonas pode ser utilizado na isozona de interesse.

É importante ressaltar que, devido ao pequeno tamanho da série de dados pluviográficos da estação de Aiuaba, CE, não foi possível a determinação dos coeficientes para outros períodos de retorno. No entanto, os resultados obtidos fornecem um indicativo de confiabilidade na utilização dessa técnica. Como o método das isozonas é comumente empregado para subsidiar a construção de pequenas obras (Fechine Sobrinho, 2011) esses resultados reforçam a hipótese de que o uso do método pode ser assegurado, com certa margem de confiança, em locais desprovidos de dados pluviográficos desde que, seja conduzida uma análise de validação.

A avaliação das cinco distribuições de probabilidade testadas para as estações de Juazeiro do Norte, Crato e Barbalha, no Ceará encontram-se na Tabela 3. Os valores teóricos críticos a nível de significância de $5 \%$, para o teste $t$ do coeficiente angular foram de 2,03 e para os testes de aderência de Lilliefors e qui-quadrado $\left(\chi^{2}\right)$ foram de, respectivamente, 0,146 e 9,49 . Observa-se que a hipótese nula deixou de ser rejeitada em todas as distribuições avaliadas tanto pelo teste $\chi^{2}$ quanto pelo de Lilliefors indicando que os dados se ajustam às distribuições testadas.

$\mathrm{O}$ teste $\mathrm{t}$ apresentou como resultados, para todas as distribuições, estatísticas de teste superiores ao valor crítico; desta forma, a hipótese nula de que o coeficiente angular é zero foi rejeitada para todas as distribuições indicando que os valores observados e esperados se ajustam a uma reta.

Para a estação de Juazeiro do Norte, CE, os menores valores de $\chi^{2}$ foram obtidos para as distribuições Gama II e III, sendo que na Gama III se verificaram DQM e DPMA menores e a estatística de teste $t$ apresentou o maior valor. Desta forma, considerou-se que os dados dessa estação se ajustam melhor à distribuição Gama III. Seguindo uma avaliação semelhante para as estações de Crato, CE, e Barbalha, CE, foram escolhidas, respectivamente, as distribuições Log. Normal III e Gama II. Mesmo com as escolhas dessas distribuições utilizando coeficientes, índices e testes de aderência, não se constatou uma diferença significativa entre as distribuições avaliadas corroborando com os resultados de Elsebaie (2012) que avaliaram distintos modelos estatísticos e não encontraram diferenças significativas de desempenho. Tais resultados podem indicar a influência da similaridade climática da região das estações ou ainda da utilização nos ajustes de séries pluviométricas com longos registros de observações (Svensson et al., 2007). Portanto, a escolha de um ou outro modelo estatístico de probabilidade para representar a região

Tabela 3. Intervalo de $95 \%$ de confiança para o coeficiente angular (a \pm Erro); intervalo de $95 \%$ de confiança para intercepto $\mathrm{Y}\left(\mathrm{b} \pm\right.$ Erro); coeficiente de determinação $\left(\mathrm{R}^{2}\right)$; desvio quadrático médio $(\mathrm{DQM})$; desvio percentual médio (DPMA); resultado dos testes de aderência quí-quadrado $\left(\chi^{2}\right)$; Lilliefors (L) de diferentes distribuições de probabilidades para as estações avaliadas e estatística de teste $\mathrm{t}\left(\mathrm{t}_{0}\right)$ do coeficiente angular

\begin{tabular}{|c|c|c|c|c|c|c|c|c|c|}
\hline Estação & Função & $a \pm E r r o$ & b \pm Erro & $\mathbf{R}^{2}$ & DQM & DPMA & $\chi^{2}\left(^{*}\right)$ & Lilliefors (*) & $t_{0}$ \\
\hline \multirow{5}{*}{ Juazeiro do Norte-CE } & Gumbel & $1,06 \pm 0,04$ & $-0,03 \pm 0,03$ & 0,99 & 0,17 & 10,06 & 4,56 & 0,10 & 48,89 \\
\hline & Log. Normal II & $1,05 \pm 0,04$ & $-0,02 \pm 0,02$ & 0,99 & 0,22 & 9,93 & 3,91 & 0,09 & 49,61 \\
\hline & Log. Normal III & $1,05 \pm 0,05$ & $-0,03 \pm 0,03$ & 0,98 & 0,18 & 13,38 & 5,71 & 0,09 & 42,89 \\
\hline & Gama II & $1,01 \pm 0,04$ & $0,00 \pm 0,02$ & 0,99 & 0,23 & 10,85 & 2,82 & 0,08 & 48,71 \\
\hline & Gama III & $1,02 \pm 0,04$ & $-0,01 \pm 0,02$ & 0,99 & 0,18 & 9,87 & 3,03 & 0,07 & 52,10 \\
\hline \multirow{5}{*}{ Crato-CE } & Gumbel & $1,04 \pm 0,04$ & $-0,03 \pm 0,02$ & 0,99 & 0,15 & 11,17 & 6,19 & 0,11 & 52,97 \\
\hline & Log. Normal II & $1,02 \pm 0,03$ & $-0,02 \pm 0,02$ & 0,99 & 0,12 & 9,16 & 9,20 & 0,10 & 59,73 \\
\hline & Log. Normal III & $1,03 \pm 0,03$ & $-0,01 \pm 0,02$ & 0,99 & 0,12 & 9,04 & 4,38 & 0,10 & 61,44 \\
\hline & Gama II & $1,02 \pm 0,03$ & $-0,01 \pm 0,02$ & 0,99 & 0,10 & 8,28 & 2,57 & 0,10 & 62,08 \\
\hline & Gama III & $1,01 \pm 0,04$ & $0,00 \pm 0,02$ & 0,99 & 0,11 & 8,13 & 6,27 & 0,10 & 58,50 \\
\hline \multirow{5}{*}{ Barbalha-CE } & Gumbel & $1,08 \pm 0,05$ & $-0,04 \pm 0,03$ & 0,98 & 0,16 & 13,27 & 1,17 & 0,09 & 40,77 \\
\hline & Log. Normal II & $1,04 \pm 0,05$ & $-0,02 \pm 0,03$ & 0,98 & 0,14 & 9,27 & 1,13 & 0,08 & 46,25 \\
\hline & Log. Normal III & $1,05 \pm 0,05$ & $-0,02 \pm 0,03$ & 0,98 & 0,13 & 8,21 & 1,08 & 0,06 & 45,78 \\
\hline & Gama II & $1,02 \pm 0,04$ & $-0,01 \pm 0,03$ & 0,98 & 0,13 & 8,99 & 1,10 & 0,07 & 47,64 \\
\hline & Gama III & $1,02 \pm 0,04$ & $-0,01 \pm 0,03$ & 0,98 & 0,13 & 8,59 & 2,06 & 0,06 & 47,40 \\
\hline
\end{tabular}

\footnotetext{
* Nível de significância dos testes: 0,05
} 
dependerá do rigor das estimativas e da praticidade de uso (Alhassoun, 2011).

As funções $\chi^{2}=\mathrm{f}(\mathrm{s})$ utilizadas na determinação do parâmetro "s" das equações IDF estão representadas na Figura 3. Para as três curvas os ajustes polinomiais de " $s$ " no intervalo $[-4,5 ;-1) \mathrm{e}$ lineares no intervalo $[-1 ; 4)$, comprovam que o valor de "s" ótimo encontra-se no intervalo de definição das funções polinomiais. Neste contexto os valores de "s" que minimizam essas funções são de $-2,75 ;-2,78$ e -2,76, para as respectivas equações IDF de Juazeiro do Norte, CE, Crato, CE e Barbalha, CE (Tabela 3).

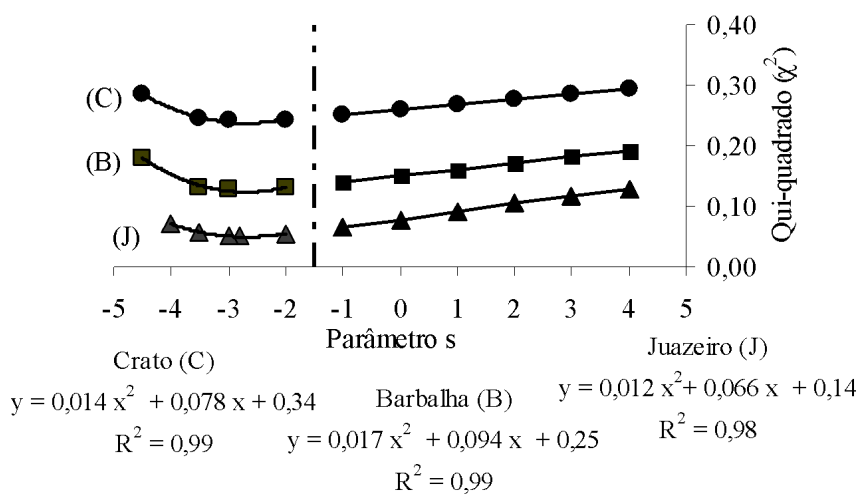

Figura 3. Representação do parâmetro s em função dos valores de Qui-quadrado

Os valores dos coeficientes e as equações IDF desenvolvidas a partir dos dados gerados pelo método das isozonas, válidos para durações inferiores a 240 min e período de retorno de até 100 anos, estão na Tabela 4.

Tabela 4. Parâmetro dos modelos IntensidadeDuração-Frequência (IDF) para estações avaliadas

\begin{tabular}{|c|c|c|c|c|c|c|}
\hline \multirow{2}{*}{ Estação } & \multicolumn{5}{|c|}{ Parâmetros } & \multirow{2}{*}{ Equação } \\
\hline & a & b & C & $n$ & $s$ & \\
\hline $\begin{array}{l}\text { Juazeiro } \\
\text { do Norte }\end{array}$ & 28,337 & 0,104 & 10,845 & 0,813 & $-2,750$ & $\mathrm{I}=\frac{28,337(\operatorname{Tr}-2,75)^{0,104}}{(\mathrm{t}+10,845)^{0,813}}$ \\
\hline Crato & 20,156 & 0,083 & 5,400 & 0,726 & $-2,786$ & $\mathrm{I}=\frac{20,156(\operatorname{Tr}-2,786)^{0,083}}{(\mathrm{t}+5,4)^{0,726}}$ \\
\hline Barbalha & 21,993 & 0,092 & 6,825 & 0,750 & $-2,765$ & $\mathrm{I}=\frac{21,993(\operatorname{Tr}-2,765)^{0,092}}{(\mathrm{t}+6,825)^{0,75}}$ \\
\hline
\end{tabular}

A aplicação das três equações da Tabela 4 resulta em valores idênticos de intensidades para quaisquer valores de duração e período de retorno. Este fato está relacionado à proximidade geográfica entre as estações estando os municípios de Juazeiro, Crato e Barbalha situados na mesma zona climática, com limites geográficos muito próximos e regimes pluviométricos anuais similares (Fechine Sobrinho, 2011).

\section{Conclusões}

1. A validação do método das isozonas para as estações localizadas em zonas climatológicas distintas da Região Metropolitana do Cariri (RMC) mostrou que as intensidades máximas estimadas pelo método foram confiáveis quando comparadas as estimadas pelas equações IDF. Nas regiões de mesma zona climatológica da RCM os erros entre as relações pluviométricas $1 \mathrm{~h} / 24 \mathrm{~h}$ e $6 \mathrm{~min} / 24 \mathrm{~h}$ fornecidas pelas isozonas e as obtidas pelos dados pluviográficos foram inferiores a $2 \%$.

2. As precipitações máximas diárias anuais das estações de Juazeiro do Norte, CE, Crato, CE e Barbalha, CE, utilizadas na aplicação do método das isozonas se ajustaram respectivamente às distribuições Gama III, Log-Normal II e Gama II.

3. As equações de Intensidade-Duração-Frequênica determinadas para os municípios de Juazeiro, Crato e Barbalha, fornecem valores calculados de intensidades semelhantes entre si devido à proximidade geográfica entre as estações que integram a mesma zona climatológica.

\section{Agradecimentos}

Ao Professor Dr. José Carlos de Araújo, do grupo de Estudos Hidrossedimentológicos do Semiárido - HIDROSED, pela concessão dos dados pluviográficos da estação de Aiuaba, CE.

\section{Literatura Citada}

Alhassoun, S. A. Developing an empirical formulae to estimate rainfall intensity in Riyadh region. Journalof King SaudUniversity, v.23, p.81-88, 2011.

Back, A. Seleção de distribuição de probabilidade para chuvas diárias extremas do estado de Santa Catarina. Revista Brasileira de Meteorologia, v.16, p.211-222, 2001.

Back, A. Relações entre precipitações intensas de diferentes durações ocorridas no município de Urussanga, SC. Revista Brasileira Engenharia Agrícola e Ambiental, v.13, p.170175, 2009.

Back, A. J.; Oliveira. J. L. R.; Henn, A. Duration-Frequency relationships of heavy rainfall in Santa Catarina, Brazil. Revista Brasileira de Ciências do Solo, v.36, p.1015-1022, 2012.

Camargo, A. P.; Sentelhas, P. C. Avaliação do desempenho de diferentes métodos de estimativas de evapotranspiração potencial no Estado de São Paulo, Brasil. Revista Brasileira de Agrometeorologia, v.5, p.89-97, 1997.

Catalhunha, M. J.; Sediyama, G. C.; Leal, B. G.; Soares, C. P. B.; Ribeiro, A. Aplicação de cinco funções densidade de probabilidade a séries de precipitação pluvial no Estado de Minas Gerais. Revista Brasileira de Agrometeorologia, v.10, p.153-162, 2002.

Elsebaie, I. H. Developing rainfall intensity-duration-frequency relationship for two regions in Saudi Arabia. Journal of King Saud University, v.24, p.131-140, 2012.

Fechine Sobrinho, V. Aplicação do método das isozonas na obtenção das equações IDF de chuvas intensas dos municípios de Juazeiro do Norte, Barbalha e Crato - CE. Fortaleza: UFC, 2011. 72p. Dissertação Mestrado

Garcia, S. S.; Amorim, R. S. S.; Couto, E. G.; Stopa, W. H. Determinação da equação intensidade-duração-frequência para três estações meteorológicas do Estado do Mato Grosso. Revista Brasileira de Engenharia Agrícola e Ambiental, v.15, p.575-581, 2011. 
Genovez, A. M.; Zuffo, A. C. Chuvas intensas no Estado de São Paulo: estudos existentes e análise comparativa. Revista Brasileira de Recursos Hídricos, v.5, p.45-58, 2000.

Laura, A. A. Estudo do uso de séries parciais e anuais na análise de freqüência de chuvas intensas aplicando modelos de distribuição de probabilidade. Fortaleza: UFC, 1997. 224p. Dissertação Mestrado

Martins, S. G.; Avanazi, J. C.; Silva, M. L. N.; Curi, N.; Norton, L. D.; Fonseca, S. Rainfall erosivity and rainfall return period in the experimental watershed of Aracruz, in the Coastal Plain of Espírito Santo, Brazil. Revista Brasileira Ciências Solo, v.34, p.999-1004, 2010.

Oliveira, L. F. C.; Antonini, J. C. A.; Fioreze, A. P.; Silva, M. A. $\mathrm{S}$. Métodos de estimativa de precipitação máxima para o Estado de Goiás. Revista Brasileira de Engenharia Agrícola e Ambiental, v.12, p.620-625, 2008.
Rodrigues, J. O.; Andrade, E. M.; Oliveira, T. S.; Lobato, F. A. O. Equações de Intensidade-Duração e Freqüência de chuvas para as localidades de Fortaleza e Pentecoste, Ceará. Scientia Agraria, v.9, p.511-519, 2008.

Santos, G. G.; Griebeler, N. P.; Oliveira, L. F. C. Chuvas intensas relacionadas à erosão hídrica. Revista Brasileira Engenharia Agrícola e Ambiental, v.14, p.115-123, 2010.

Svensson, C.; Clarke, R. T.; Jones, D. A. An experimental comparison of methods for estimating rainfall intensityduration-frequency relations from fragmentary records. Journal of Hydrology, v.341, p.79-89, 2007.

Torrico, J. J. T. Práticas Hidrológicas. Rio de Janeiro: Transcon, 1974. 119p.

Wilken, P. S. Engenharia de drenagem superficial. São Paulo: CETESB, 1978. 478p. 